\title{
Behavioral contrast and responding during multiple food-food, food-water, and water-water schedules
}

\author{
R. H. ETTINGER and F. K. McSWEENEY \\ Washington State University, Pullman, Washington 99164
}

\begin{abstract}
Five pigeons pecked lighted keys for food reinforcers delivered by several multiple variable interval 2-min variable interval 2-min schedules. At different times, the components of the multiple schedule both supplied food reinforcers, both supplied water, or one supplied food and the other supplied water. Rates of responding during the water component of the food-water schedule were lower than the rates during comparable components of the water-water schedules (negative contrast). But, the rates of responding during the food component of the food-water schedule were not greater than the rates of responding during comparable components of the foodfood schedules (absence of positive contrast) at two different levels of water deprivation. These results raise questions about several theories of behavioral contrast, and they may restrict the scope of any theory that attributes positive and negative contrast to symmetrical factors.
\end{abstract}

Recently, interest has focused on the behavioral contrast that occurs when subjects respond on multiple schedules. Contrast has been defined in different ways (McSweeney \& Norman, 1979), but it usually refers to changes in the rate of responding during one, constant, component of the multiple schedule with changes in the rate of responding or conditions of reinforcement during the other, variable, component. Increases in the rate of responding during the constant component with decreases in the rate of responding or conditions of reinforcement in the variable component are labeled positive contrast. Decreases in the rate of responding during the constant component with increases in the variable rate of responding or conditions of reinforcement are labeled negative contrast.

The present paper addresses two questions about behavioral contrast. First, several authors have asked whether positive and negative contrast are produced by symmetrical mechanisms. Herrnstein (1970) and Rachlin (1973) have proposed theories that suggest that they are, but Schwartz (1975) has argued that they need not be. To date, two studies appear to support Schwartz's argument by finding one type of contrast under conditions that do not produce the other. McSweeney (1978) found negative, but not positive, contrast when pigeons pressed treadles for food reinforcers. Schwartz (1975) found that moving the stim-

These experiments are part of a dissertation submitted to the Department of Psychology, Washington State University, by Richard H. Ettinger, in partial fulfillment of the requirements for the PhD degree. Portions of these data were presented at the 1979 meeting of the Psychonomic Society in Phoenix, Arizona. Reprints may be obtained from Frances K. McSweeney, Department of Psychology, Washington State University, Pullman, Washington 99164. uli that signaled the components of the multiple schedule changed positive, but not negative, contrast. These results do not unequivocally support Schwartz's theory, however. McSweeney (1978) argued that they can be reconciled with a symmetrical theory.

Second, questions have arisen about the conditions that produce contrast (e.g., Rachlin, 1973). Although different studies may support slightly different conclusions, many studies have reported contrast when the characteristics of one particular reinforcer, such as its size (e.g., Hamilton \& Silberberg, 1978) or rate (e.g., Reynolds, 1961), are manipulated. But, few studies have asked whether contrast occurs when the quality or type of reinforcer obtained from the variable component changes. Some studies have reported contrast with an instrumental response when both the rate and type of reinforcer presented during the other component varied (Beninger, 1972; Beninger \& Kendall, 1975; Premack, 1969; Woodruff, 1979). But, only one experiment reported contrast that could be clearly attributed to changes in the quality of the reinforcer alone (Ettinger, McSweeney, \& Norman, 1981).

Ettinger et al. (1981) found both positive and negative contrast when the type of grain provided by a component of a multiple schedule varied. They defined positive contrast as an increase in the rate of responding during a constant component that provided a moderately preferred grain (wheat) when a less preferred grain (oats) was substituted for the moderately preferred one during the variable component. Negative contrast was defined as a decrease in the rate of responding for the moderately preferred grain when a highly preferred grain (split peas or mixed grain) was substituted for the moderately preferred one in 
the variable component. The subjects' preferences for the three grains were determined by the amount eaten in home-cage preference tests.

Ettinger et al. (1981) reported both positive and negative contrast when the type of grain used as reinforcer changed. But, no data indicate whether other types of qualitative changes produce contrast. Several authors have argued recently that different types of qualitative changes may change behavior differently (e.g., Hursh, 1980; Rachlin, Kagel, \& Battalio, 1980). Drawing on economic theory, these theorists distinguish between reinforcers that are substitutable for each other (e.g., different rates, amounts, or types of food) and reinforcers that are not substitutable (e.g., food and water). The details of their definitions of substitutability are beyond the scope of this paper, but, if substitutability is an important psychological concept, then qualitative changes in the reinforcer produced by changing the type of grain, a substitutable change, might not affect behavior in the same way as would changing the reinforcer from food to water, a nonsubstitutable change.

The present study asks whether contrast occurs when the quality of a reinforcer is manipulated by changing the reinforcer presented during the variable component from food to water or vice versa. Contrast is defined according to the intraschedule definition (McSweeney \& Norman, 1979) used in the Ettinger et al. (1981) study. That is, this study compares the rate of responding during a baseline schedule that supplied the same rates of the same reinforcer in both components with the rates of responding during a heterogeneous schedule that supplies the same rates of different reinforcers in the two components. Positive contrast occurs when the rate of responding for a reinforcer during the heterogeneous schedule exceeds that for the same reinforcer during the baseline schedule. Negative contrast occurs when the rate of responding for a reinforcer during the heterogeneous schedule is less than that for the same reinforcer during the baseline schedule.

\section{EXPERIMENT 1}

\section{Method}

Subjects. Five experimentally naive White Carneaux pigeons, maintained at $85 \%$ to $90 \%$ of their free-feeding and free-drinking body weights, were used as subjects. Deprivations were established by withholding both food and water until the desired weights were achieved. Then, stable weights were maintained by supplemental feeding and by 5 min access to water immediately following each daily experimental session. The same deprivations were main- tained throughout all six phases of the experiment. These deprivations were selected because pilot research showed that they supported responding for both food and water.

Apparatus. The experimental chamber was a modified GrasonStadler three-key pigeon chamber (Model 1122) enclosed in a sound-attenuating box. The two outer keys, which signaled the alternating variable interval ( $\mathrm{VI}$ ) schedules, were separated by $14.5 \mathrm{~cm}$ center to center. The center key was removed. Access to the grain hopper was through a $4.5 \times 5 \mathrm{~cm}$ opening located centrally, $6 \mathrm{~cm}$ above the floor. Access to the water dipper was through a $3 \times 4 \mathrm{~cm}$ opening located $4 \mathrm{~cm}$ from the lower right corner of the panel. The water dipper was a solenoid-operated arm that raised a $1-\mathrm{ml}$ cup from a reservoir containing tap water to the opening in the panel. A shielded houselight, located $6.5 \mathrm{~cm}$ to the left of and $4 \mathrm{~cm}$ above the left key, was continuously illuminated. A ventilation fan provided masking noise. All of the experimental events were programmed and recorded by conventional electromechanical equipment located in an adjacent room.

Procedures. The subjects were shaped to peck a response key by a successive approximations procedure. After they responded at a high, steady rate for continuous food reinforcement, they were trained on VI 15-sec, VI 30-sec, and mult VI 2-min VI 2-min schedules of food reinforcement. The subjects were then trained to peck a key for water by a similar procedure. For the first session, they were given $3 \mathrm{ml}$ of a saline solution $1 \mathrm{~h}$ before shaping began to increase the probability of responding for water. Shaping with both food and water was completed before the experiment began.

When the experiment began, the subjects always responded on mult VI 2-min VI 2-min schedules. The schedules delivered reinforcers according to a 12 -interval series constructed according to a procedure described by Catania and Reynolds (1968, Appendix II). A red light appeared on the left key during one component of the multiple schedule, and a blue light appeared on the right key during the other. The key lights alternated every $60 \mathrm{sec}$. They were extinguished for $4 \mathrm{sec}$ during the presentation of a reinforcer, but the component timer did not stop. Reinforcers that became available but were not collected during a component were held over for the next presentation of that component. A two-key procedure was used because two-manipulanda schedules may facilitate the appearance of contrast (e.g., McSweeney, 1978).

The experiment consisted of six phases. During the first and third phases, pecks produced food reinforcers during both components of the multiple schedule. During the fourth and sixth phases, water was the reinforcer in both components. During the second and fifth phases, food was the reinforcer in one component and water was the reinforcer in the other. Food reinforcers were $4 \mathrm{sec}$ access to a magazine containing mixed grain. The magazine was illuminated with white light during reinforcement. Water reinforcers were $4 \mathrm{sec}$ access to a dipper containing approximately $1 \mathrm{ml}$ of water. A white light illuminated the area around the water dipper when water was available. Table 1 summarizes the reinforcers presented during each phase of the experiment and the key from which they were obtained.

Subjects responded to each condition until response rates stabilized. Responding was considered to be stable for each subject when the rates of responding during the last five sessions of each condition all fell within the range of the rates of responding during previous sessions of the same condition. Responding was considered to be stable only when the performances of all birds, responding during each component, met this criterion. Phases 1 to 6 were presented for $33,16,21,24,18$, and 25 sessions, respectively.

Table 1

Summary of the Reinforcers Presented During Each Component of the Multiple Schedule in Each Phase of Experiment 1

\begin{tabular}{ccccccc}
\hline Component & Phase 1 & Phase 2 & Phase 3 & Phase 4 & Phase 5 & Phase 6 \\
\hline Left Key & Food & Food & Food & Water & Water & Water \\
Right Key & Food & Water & Food & Water & Food & Water \\
\hline
\end{tabular}


An additional session, following the last session of each phase, was conducted to obtain videotape records of the topography of responding. Response rates during these sessions were not computed.

Sessions were conducted daily, six to seven times per week. They began when the subject emitted its first peck and terminated after 20 reinforcers had been delivered. Observation of the subjects indicated that they collected all reinforcers during each phase of the experiment and insured that the rates of reinforcement obtained from each component did not change from phase to phase.

\section{Results and Discussion}

Table 2 presents the mean rates of responding for each subject during each component of each multiple schedule. Means were calculated over the last five sessions for which each schedule was available. Rates of responding were calculated by dividing the number of responses during a component by the time for which that component was available. The time of availability for water reinforcers was considered to be $4 \mathrm{sec}$.

Table 2 suggests that the rate of responding for constant food reinforcers did not change systematically when water was substituted for food in the variable component (Phases 1 to 3). The average rate of responding during the food component of the foodwater schedule was greater than the rate during the corresponding component of the food-food baselines in 3 of 10 comparisons and less than this rate in 7 of 10 comparisons. A one-way repeated measures analysis of variance applied to the rates of responding during the constant food components in Phases 1,2 , and 3 confirms the visual impression. It was not significant $[F(2,8)=.66, p>.05]$.

Table 2 also suggests that the rate of responding for a constant water reinforcer did decrease systematically when food was substituted for water in the variable component (Phases 4 to 6). The average rate of responding during the water component of the food-water schedule (Phase 5) was less than the average rate of responding during the corresponding component of the water-water schedules (Phases 4 and 6) in 9 of 10 comparisons. A one-way repeated measures analysis of variance, applied to the rates of responding during the constant water components in Phases 4, 5, and 6, confirms this impression. It was statistically significant $[F(2,8)=5.38, p<.05]$.
Matched $t$ tests also showed that this significant change was produced by the experimental manipulation rather than by fluctuations in responding over time. The rate of responding during the water component in Phase 5 was significantly less than the rate during the same component in both Phase $4[\mathrm{t}(4)=$ $2.44, \mathrm{p}<.05]$ and Phase $6[\mathrm{t}(4)=5.88, \mathrm{p}<.01]$.

It is tempting to describe these results as the absence of positive contrast under conditions that did produce negative contrast. An increase in responding that could be labeled positive contrast did not occur during the constant food component when the reinforcer available from the other component changed from food to water. But, the rate of responding for a constant water reinforcer did decrease when food was substituted for water. This decrease fits the definition of negative contrast given earlier: It represents a decrease in the rate of responding for a constant reinforcer, with changes in the reinforcer available from the other component. The decrease was also generally opposite in direction to the change in response rate in the variable component: The rate of responding for food in Phase 5 was greater than that for water in Phases 4 and 6 in 8 of 10 comparisons. A one-way repeated measures analysis of variance showed that this change was marginally significant $[F(2,8)=3.99, .05<p<.10]$.

Finding negative contrast under conditions that failed to produce positive contrast may be puzzling for some symmetrical theories of contrast, unless it can be dismissed as a procedural artifact. One possible procedural explanation for the failure to find contrast in Phases 1 to 3 is the subjects' lack of experience with the food-water schedules. Positive contrast may have failed to occur if it required some time to develop. Negative contrast was observed during Phase 5, which was conducted after the subjects had had substantial experience responding on food-water schedules. Positive contrast might have been observed if the subjects had had as much experience with the schedules when it was studied.

A second possible procedural explanation is that the change of reinforcers when water was substituted for food was not large enough to produce positive contrast. For example, it has been argued that con-

Table 2

Mean Rates of Responding Emitted by Each Subject Responding on Each Component of Each Multiple Schedule for the Last Five Sessions for Which That Schedule Was Available

\begin{tabular}{|c|c|c|c|c|c|c|c|c|c|c|c|c|}
\hline \multirow[b]{2}{*}{ Subject } & \multicolumn{2}{|c|}{ Phase 1} & \multicolumn{2}{|c|}{ Phase 2} & \multicolumn{2}{|c|}{ Phase 3} & \multicolumn{2}{|c|}{ Phase 4} & \multicolumn{2}{|c|}{ Phase 5} & \multicolumn{2}{|c|}{ Phase 6} \\
\hline & Food & Food & Food & Water & Food & Food & Water & Water & Food & Water & Water & Water \\
\hline 902 & 29.2 & 33.0 & 25.8 & 25.3 & 21.9 & 31.5 & 19.9 & 36.9 & 25.8 & 12.8 & 21.9 & 40.7 \\
\hline 5964 & 55.6 & 51.3 & 55.1 & 35.2 & 57.8 & 51.6 & 36.8 & 44.7 & 53.9 & 29.7 & 33.8 & 51.1 \\
\hline 7642 & 56.3 & 58.3 & 37.1 & 51.4 & 45.5 & 71.2 & 21.4 & 39.7 & 45.1 & 48.5 & 50.2 & 98.2 \\
\hline 5258 & 32.0 & 58.2 & 35.4 & 40.6 & 44.3 & 59.6 & 49.7 & 67.6 & 55.6 & 28.0 & 62.7 & 52.8 \\
\hline 5974 & 33.1 & 38.7 & 46.8 & 48.8 & 59.1 & 36.1 & 37.2 & 55.0 & 78.1 & 23.5 & 45.1 & 48.9 \\
\hline Mean & 41.2 & 47.9 & 40.0 & 40.3 & 45.7 & 50.0 & 33.0 & 48.8 & 51.7 & 28.5 & 42.7 & 58.3 \\
\hline
\end{tabular}


trast occurs only when the components of the multiple schedule differ in value (e.g., Rachlin, 1973). According to this theory, positive contrast occurs when the constant component is more valuable than the variable component. Negative contrast occurs when the constant component is less valuable. If this is true, then the value of food may not have been sufficiently greater than the value of water in Phase 2 to produce positive contrast.

In support of this explanation, some evidence suggests that food and water may not have differed much during Phases 1 to 3. A one-way repeated measures analysis of variance, applied to the rates of responding during the variable component in Phases 1,2 , and 3 , was not significant $[F(2,8)=2.27, p>.05]$, even though the reinforcer supporting that responding was sometimes food and sometimes water. Admittedly, similarities or differences in the absolute rates of responding for food and water would not unequivocably reflect similarities or differences in reinforcer value, because the topography of the response may also change with changes in the reinforcers (Jenkins \& Moore, 1973). But, the failure to find a significant change in response rates with changes in the reinforcer is suggestive enough to require further study.

\section{EXPERIMENT 2}

Experiment 2 tested these two procedural explanations for the results of Experiment 1. It was conducted after Experiment 1, when the subjects had had more experience responding on food-water schedules. It was assumed that food could be made more valuable relative to water by changing the subjects' deprivation for water. A deprivation for water was selected that represented the least possible deprivation at which subjects would consume all of the reinforcers collected during an experimental session. It was reasoned that the value of water could not decrease further without allowing any contrast that occurred to be attributed to changes in the rate of reinforcement, rather than to changes in their quality.

\section{Method \\ Subjects. The same subjects served in this experiment as in Ex- periment 1, except for Subject 5974, which became sick during the experiment. The subjects were maintained at $85 \%$ to $90 \%$ of their free-feeding and free-drinking body weights by supplemental feed- ing and $30 \mathrm{~min}$ access to water immediately following each daily experimental session.}

Apparatus and Procedure. The apparatus was identical to that used in Experiment 1. The procedure was identical to that used in Phases 1, 2, and 3 of Experiment 1, presented in that order. Phases 1,2 , and 3 were conducted for 27,25 , and 20 sessions, respectively.

\section{Results and Discussion}

Table 3 presents the mean rates of responding by each subject during each component of each multiple
Table 3

Mean Rates of Responding Emitted by Each Subject Responding on Each Component of Each Multiple Schedule for the Last Five Sessions for Which That Schedule Was Available

\begin{tabular}{|c|c|c|c|c|c|c|}
\hline \multirow[b]{2}{*}{ Subject } & \multicolumn{2}{|c|}{ Phase 1} & \multicolumn{2}{|c|}{ Phase 2} & \multicolumn{2}{|c|}{ Phase 3} \\
\hline & Food & Food & Food & Water & Food & Food \\
\hline 902 & 21.2 & 31.3 & 24.0 & 6.4 & 28.6 & 44.3 \\
\hline 5964 & 51.0 & 61.1 & 58.3 & 40.5 & 56.8 & 64.9 \\
\hline 7642 & 47.9 & 106.8 & 55.4 & 33.0 & 50.3 & 117.2 \\
\hline 5258 & 68.9 & 70.5 & 84.9 & 24.5 & 83.8 & 72.8 \\
\hline Mean & 47.3 & 67.4 & 55.7 & 26.1 & 54.9 & 74.8 \\
\hline
\end{tabular}

schedule. Rates of responding were calculated as they were for Table 2 .

Table 3 suggests that the present procedure did increase the difference between the values of food and water. A one-way repeated measures analysis of variance, applied to the rates of responding during the variable component in Phases 1,2 , and 3, was statistically significant $[F(2,6)=12.98, p<.01]$. Matched $t$ tests also showed that the significant change was produced by the experimental manipulation rather than by fluctuations in response rates over time: The rate of responding for water during the variable component in Phase 2 was significantly less than the rate for food in both Phases $1[\mathrm{t}(3)=3.40, \mathrm{p}<.05]$ and $3[\mathrm{t}(3)=3.80, \mathrm{p}<.05]$.

Again, however, positive contrast did not occur. The rates of responding did change across phases. Responding during the food component of the foodwater schedule was greater than the responding during the corresponding component of the food-food baseline in seven of eight comparisons, and a repeated measures analysis of variance, applied to the rates of responding during the constant components in Phases 1,2 , and 3 , was statistically significant $[F(2,6)=6.96, p<.05]$. But, further statistical tests suggest that these changes in the rates of responding represented fluctuations in responding across phases rather than positive contrast. $T$ tests for matched pairs showed that the rates of responding during the constant component of Phase 2 differed significantly from those during the corresponding component of the surrounding baseline schedules when the baseline was Phase $1[\mathrm{t}(3)=3.05, \mathrm{p}<.05]$, but not when it was Phase $3[\mathrm{t}(3)=.39, \mathrm{p}>.05]$. Responding during both the constant $[\mathrm{t}(3)=2.89, \mathrm{p}<.05]$ and the variable components $[\mathrm{t}(3)=2.87, \mathrm{p}<.05]$ also differed significantly between the two baseline conditions presented during Phase 1 and Phase 3. Therefore, these results are consistent with the hypothesis that the increase in responding from Phase 1 to Phase 2 represented an increase in responding over time. They are not consistent with the hypothesis that the increase in responding represented positive contrast.

Figure 1 illustrates behavioral contrast across sessions for both experiments. The three sets of five 


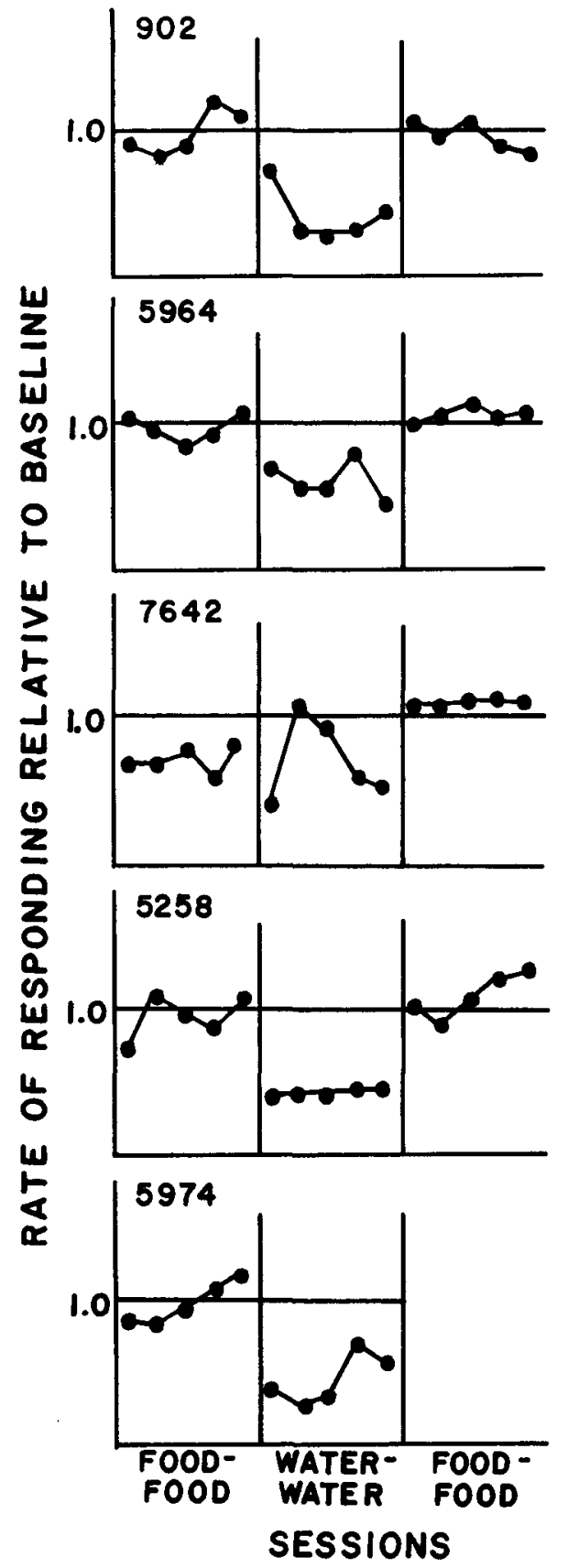

Figure 1. Rates of responding relative to baseline rates of responding for exch session during which the food-water schedules were presented. Points plotted on the left side of the figure were calculated by dividing the rate of responding during the food component of the food-water schedule presented in Phase 2 of Experiment 1 by $a$ baseline rate of responding for food. Baseline rates were calculated by averaging the five session means of the rates of responding during the same components in Phases 1 and 3. Points on the right side of the figure were calculated in the same way for Experiment 2. Points in the middle of the figure were calculated by dividing the rate of responding during the water component of the food-water schedule presented in Phase 5 of Experiment 1 by a baseline rate of responding for water. Baseline rates of responding were calculated by averaging the five session means of the rates of responding during the same components in Phases 4 and 6. Points that fall above the horizontal line at 1.0 may represent positive contrast; points that fall below the line may represent negative contrast. points, plotted for each subject, represent the rates of responding by that subject during the constant component of the food-water schedules presented during Phases 2 and 5 of Experiment 1 and during Phase 2 of Experiment 2, respectively. These points have been plotted relative to a baseline rate of responding obtained by averaging the rates in the same component during the two surrounding baseline multiple schedules. The rates that were averaged were the five session means taken from Tables 2 and 3 for each subject. Points that fall above the line at 1.0 in the first and third sets of points may represent positive contrast; points that fall below the line in the second set may represent negative contrast. Figure 1 clearly shows that negative, but not positive, contrast occurred over individual sessions, as well as for the average rates of responding.

The failure to observe contrast in Experiment 2 casts doubt on the two procedural explanations for the failure to observe positive contrast in Experiment 1 . It seems unlikely that the food and water reinforcers did not differ enough to produce contrast. The differences between the reinforcers seemed to be more extreme in Experiment 2 than they were in Experiment 1, but contrast still did not occur. It also seems unlikely that conducting the experiment for too few sessions explained the failure to observe contrast. Positive contrast failed to occur during Experiment 2 when subjects responded on the foodwater schedules for 25 sessions during the experiment and 59 sessions overall. The relatively quick development of negative contrast within the 16 sessions of Phase 5 of Experiment 1 makes it seem unlikely that more experience would have produced positive contrast.

\section{GENERAL DISCUSSION}

Responding for a constant water reinforcer decreased when the reinforcer presented in the other component changed from water to food, but responding for a constant food reinforcer did not increase when the reinforcer presented in the other component changed from food to water. This failure to find an increase in responding for food is particularly surprising because the availability of water might make the food itself more valuable. An increase in the value of food should have produced an increase in response rates, which would make the reporting of positive contrast even more likely, but this did not occur. The unexpectedness of these results raises the question of whether they are an artifact of a procedural detail. Several additional procedural explanations may be considered.

First, a ceiling effect probably did not interfere with the observation of positive contrast. The increase in responding that represents contrast would not be expected if subjects were responding as fast as they could before the contrast manipulation oc- 
curred. But, this is unlikely because the present subjects responded at 40 to 50 responses/min during the constant components of the baseline food-food schedule presented in Phase 1 of both experiments. Forty responses per minute represents only a moderate rate of keypecking for pigeons, in general, and these subjects, in particular, showed much higher rates of responding (almost 75 responses/min on the average) later in the experiment. Therefore, it is unlikely that a ceiling effect interfered with contrast.

Second, failures to discriminate between the components probably did not produce the failure to observe contrast. The rates of responding during the components would change in the same direction, not in the opposite direction required for contrast, if the subjects could not tell the components apart (Rachlin, 1973). But the two-key procedure was used to facilitate discrimination between the components, and videotaped observations of the subjects showed that discrimination did occur: Responses during the food component resembled eating, and those during the water component resembled drinking, as described by Jenkins and Moore (1973).

Third, the design of the apparatus probably did not produce the present results. The food hopper and water dipper were not symmetrically placed. Therefore, it might be argued that changes in the topography of responding with changes in the position of the available reinforcers might have interfered with the appearance of positive contrast. However, the required changes in the topography of responding were not apparent in the videotapes of the sessions, and an analysis of the apparatus changes that actually occurred casts further doubt on this explanation. Only the position of the variable key with respect to its reinforcer changed when contrast was observed. To attribute the present results to apparatus design, it must be argued that changing the relation between the variable key and its reinforcer somehow changed responding on the constant key when it was available. Although this is possible, it seems unlikely.

The present results should be replicated and extended to many more qualitative changes in reinforcers because they have important theoretical implications. First, the results are consistent with Schwartz's (1975) argument that positive and negative contrast may not be symmetrical. This, in turn, may imply that the symmetrical theories of contrast are either incorrect or they do not apply to the contrast produced by food and water manipulations. Restricting the scope of the symmetrical theories to exclude food and water manipulations would make the theories less general, and it would not explain the failure to find symmetrical results in the other studies described earlier (McSweeney, 1978; Schwartz, 1975). But, restricting the theories would be consistent with recent economic theories of behavior (e.g., Hursh, 1980; Rachlin et al., 1980). These theories suggest that responding for reinforcers that may be substitutable for each other, such as two different types of grain, may differ from responding for reinforcers that are not substitutable. Only further research will show whether the economic theories' distinction between substitutable and nonsubstitutable reinforcers accurately describes the conditions under which positive and negative contrast will or will not be symmetrical.

Second, the presence of negative contrast poses problems for Hearst and Jenkins' (1974) theory of negative contrast. Hearst and Jenkins argue that negative contrast occurs when subjects withdraw from a stimulus that signals the absence of reinforcers. But, attributing the present negative contrast to withdrawals from the stimulus signaling water during the food-water schedule, assumes that the subjects treated that stimulus as a signal for the absence of food rather than as a signal for the presence of water. Two considerations make this unlikely. First, the subjects made drinking movements toward the signal, possibly indicating that it was a signal for water, not for the absence of food. Second, the schedule available in the immediately preceding phase of the experiment presented water during both components. Therefore, it seems more reasonable to describe the change of schedule that produced negative contrast as the substitution of food for water during the variable component, rather than as the addition and then removal of food from the constant component of the food-water schedule. The present results, then, make Hearst and Jenkins' theory of negative contrast seem implausible.

Finally, the present results raise questions about what it means for a stimulus to be a better signal for reinforcers (Hearst \& Jenkins, 1974) or for a stimulusreinforcer dependency to exist (Gamzu \& Schwartz, 1973). Positive contrast has been said to occur when these situations exist. Intuitively, it seems that a stimulus-reinforcer dependency did exist during Phase 2 of both experiments. Food depended on the presence of one stimulus, and water depended on the presence of the other. The same might be said about stimuli that are better predictors of reinforcers. During Phase 2, one key light was a better predictor of food, while the other was a better predictor of water. In this case, positive contrast should have occurred during both components, but it did not. Explicit definitions of predictiveness or dependency should be formulated to exclude the present situations from the conditions that produce positive contrast.

\section{REFERENCES}

Beninger, R. J. Positive behavioral contrast with qualitatively different reinforcing stimuli. Psychonomic Science, 1972, 29. 307-308.

Beninger, R. J., \& Kendall, S. B. Behavioral contrast in rats 
with different reinforcers and different response topographies. Journal of the Experimental Analysis of Behavior, 1975, 24, 267-280.

Catania, A. C., \& Reynolds, G. S. A quantitative analysis of the responding maintained by interval schedules of reinforcement. Journal of the Experimental Analysis of Behavior, 1968, 11, 327-383.

Ettinger, R. H., McSweeney, F. K., \& Norman, W. D. Contrast and undermatching as a function of reinforcer duration and quality. Journal of the Experimental Analysis of Behavior, 1981, 35, 271-282.

Gamzu, E., \& Schwartz, B. The maintenance of key pecking by stimulus-contingent and response-independent food presentation. Journal of the Experimental Analysis of Behavior, 1973, 19, 65-72.

Hamilton, B. E., \& Silberberg, A. Contrast and autoshaping in multiple schedules varying reinforcer rate and duration. Journal of the Experimental Analysis of Behavior, 1978, 30, 107-122.

Hearst, E., \& Jenkins, H. M. Sign-tracking: The stimulusreinforcer relation and directed action. Austin, Tex: Psychonomic Society, 1974.

Herrnstein, R. J. On the law of effect. Journal of the Experimental Analysis of Behavior, 1970, 13, 243-266.

Hursh, S. R. Economic concepts for the analysis of behavior. Journal of the Experimental Analysis of Behavior, 1980, 34, 219-238.

Jenkins, H. M., \& Moore, B. R. The form of the auto-shaped response with food or water reinforcers. Journal of the Experimental A nalysis of Behavior, 1973, 20, 163-181.

McSweeney, F. K. Negative behavioral contrast on multiple treadle-press schedules. Journal of the Experimental Analysis of Behavior, 1978, 29, 463-473.

McSweEneY, F. K., \& Norman, W. D. Defining behavioral contrast for multiple schedules. Journal of the Experimental Analysis of Behavior, 1979, 32, 457-461.

Premack, D. On some boundary conditions of contrast. In J. Tapp (Ed.), Reinforcement and behavior. New York: Academic Press, 1969.

Rachlin, H. C. Contrast and matching. Psychological Review, $1973,80,217-234$.

Rachlin, H., Kagel, J. H., \& Battalio, R. C. Substitutability in time allocation. Psychological Review, 1980, 87, 355-374.

REYNoLDS, G. S. Behavioral contrast. Journal of the Experimental Analysis of Behavior, 1961, 4, 57-71.

Schwartz, B. Discriminative stimulus location as a determinant of positive and negative behavioral contrast in the pigeon. Journal of the Experimental Analysis of Behavior, 1975, 23, 167-176.

WooprufF, G. Behavioral contrast and type of reward: Role of elicited response topography. Animal Learning \& Behavior, $1979,7,339-346$.

(Received for publication July 10, 1980; revision accepted January 9, 1981.) 